\title{
Global Dynamics of a Compressor Blade with Resonances
}

\author{
Xiaoxia Bian, ${ }^{1,2,3}$ Fangqi Chen, ${ }^{2}$ and Fengxian $A^{1,2}$ \\ ${ }^{1}$ Department of Mechanics, Nanjing University of Aeronautics and Astronautics, Nanjing 210016, China \\ ${ }^{2}$ Department of Mathematics, Nanjing University of Aeronautics and Astronautics, Nanjing 210016, China \\ ${ }^{3}$ School of Mathematics and Physics, Yancheng Institute of Technology, Yancheng 224051, China
}

Correspondence should be addressed to Xiaoxia Bian; bxx@nuaa.edu.cn

Received 24 March 2016; Revised 16 June 2016; Accepted 12 July 2016

Academic Editor: Jaromir Horacek

Copyright (C) 2016 Xiaoxia Bian et al. This is an open access article distributed under the Creative Commons Attribution License, which permits unrestricted use, distribution, and reproduction in any medium, provided the original work is properly cited.

\begin{abstract}
The global bifurcations and chaotic dynamics of a thin-walled compressor blade for the resonant case of $2: 1$ internal resonance and primary resonance are investigated. With the aid of the normal theory, the desired form associated with a double zero and a pair of pure imaginary eigenvalues for the global perturbation method is obtained. Based on the simpler form, the method developed by Kovacic and Wiggins is used to find the existence of a Shilnikov-type homoclinic orbit. The results obtained here indicate that the orbit homoclinic to certain invariant sets for the resonance case which may lead to chaos in the sense of Smale horseshoes for the system. The chaotic motions of the rotating compressor blade are also found by using numerical simulation.
\end{abstract}

\section{Introduction}

Compressor blades are widely used in many fields of aerospace, aeronautic engineering, and mechanical industry due to their excellent mechanical properties. The problem of nonlinear dynamics of the rotating blades had attracted lots of research interest during the past decade. Various strategies and approaches have been proposed for nonlinear dynamics of rotating blades (see, e.g., [1-15]). However, theoretical analysis of global dynamics of the rotating blades has not been concerned in the current available literature. Several researchers have examined the global behaviors of plates, beams, and belt (see, e.g., [16-22]), but the results cannot be directly extended to the case of rotating blades.

Yang and Tsao investigated the vibration and stability of a pretwisted blade under nonconstant rotating speed in [1], and they also predicted the time-dependent rotating speed leads to a system with six parametric instability regions in primary and combination resonances. Surace et al. [2] dealt with the coupled bending-bending-torsion vibration of rotating pretwisted blades. Şakar and Sabuncu [3] presented the static stability and the dynamic stability of an aerofoil cross section rotating blade subjected to an axial periodic force and took into account the effects of coupling due to the center of flexure distance from the centroid, rotational speed, disk radius, and stagger angle. Al-Bedoor and AlQaisia [4] used a reduced-order nonlinear dynamic model to research the steady-state response of the rotating blade under the main shaft torsional vibration. Tang and Dowell [5] analyzed the nonlinear response of a nonrotating flexible rotor blade subjected to periodic gust excitations theoretically and experimentally. They reported that there exists a periodic or possibly chaotic behavior in the blade. Choi and Chou [6] studied the dynamic response of turbomachinery blades with general end restraints by applying the modified differential quadrature method. A Monte Carlo approach was employed to explore a supercritical Hopf bifurcation and random bifurcation of a two-dimensional nonlinear airfoil in turbulent flow by Poirel and Price [7]. Lacarbonara et al. $[8,9]$ established the governing equations of the blades under the centrifugal forces and discussed linear modal properties and the nonlinear modes of vibration away from internal resonances, respectively. Yao et al. [10] performed a nonlinear dynamic analysis of the rotating blade with varying rotating speed under high-temperature supersonic gas flow; furthermore, they [11] explored the contributions of nonlinearity, damping, and rotating speed to the steady-state nonlinear responses of the rotating blade, and they also investigated the effects of the rotating speed on nonlinear oscillations of the blade. Wang and Zhang discussed the stability 
of a spinning blade having periodically time varying coefficients for both linear model and geometric nonlinear model and obtained the stability boundary of linear model and stability of steady-state solutions of nonlinear model in [12].

In many cases, blades are usually modeled as a pretwisted, presetting, thin-walled rotating cantilever beam because the shape of the blade is very complex. Many researchers carried out studies on the dynamic behavior of the beam of this kind and obtained a lot of valuable results (see, e.g., [13-15]). Several methods have been developed to research the global bifurcation behaviors and chaotic dynamics in nonlinear systems that possess homoclinic or heteroclinic orbits. There are three methods: Melnikov method, global perturbation method, and energy-phase method. Melnikov gave the condition under which a homoclinic orbit in the unperturbation system would break under perturbation and at last lead to chaos in the system. Based on Melnikov method, Wiggins studied the global behaviors of the three basic systems [23]. Then, Kovacic and Wiggins [24] developed the global perturbation method to present Shilnikov-type homoclinic orbit for resonant system. The energy-phase method proposed by Haller and Wiggins $[25,26]$ detected the existence of single-pulse and multipulse homoclinic orbits in a class of near Hamilton systems. Applying the latter two methods, there were many applications to investigate the global behaviors (see, e.g., [16-22]).

In this paper, we obtain a sufficient condition for the existence of Shilnikov-type homoclinic orbit of a compressor blade with 2:1 internal resonance and primary resonance using normal form theory and global perturbation method. Firstly, the formulas of the simpler normal form associated with a double zero and a pair of pure imaginary eigenvalues are derived by normal form theory in Section 2. Then, the dynamics of unperturbed system and perturbed system are analyzed in Sections 3 and 4 in detail, respectively. The analysis indicate that Shilnikov-type homoclinic orbit exists in these cases. Finally, numerical simulations are given to confirm the result in Section 5 and the work ends in Section 6 with a short summary.

\section{Formulation of the Problem}

A thin-walled compressor blade of gas turbine engines with varying speed under high-temperature supersonic gas flow is considered in [11]. It is modeled as a pretwisted, presetting, thin-walled rotating cantilever beam, considering the geometric nonlinearity, centrifugal force, the aerodynamic load, and the perturbed angular speed.

The pretwisted flexible cantilever blade, with length $L$ mounted on a rigid hub with radius $R_{0}$, is considered [11]. It rotates at a varying rotating speed $\Omega(t)$ around its polar axis where $\Omega(t)=\Omega_{0}+f \cos \Omega_{1} t$, where $\Omega_{0}$ is the rotating speed at the steady-state and $f \cos \Omega_{1} t$ is a periodic perturbation. It is also allowed to vibrate flexurally in the plane making an angle $\gamma$, as shown in Figure 1(a). The rotating blade is treated as a pretwisted, presetting, thin-walled rotating cantilever beam. The length and width of the cross section of the beam in the $x$ and $y$ directions are $a$ and $b$, respectively, and the thickness of the thin-walled beam is $h$. For the purpose of describing the motion of the rotating blade, different coordinate systems are needed. The origin of the rotating coordinate system $(x, y, z)$ is located at the blade root, $x^{p}$ and $y^{p}$ are the principal axes of an arbitrary beam cross section in the local coordinates $\left(x^{p}, y^{p}, z^{p}\right)$ (Figure 1(b)), and the transformations between two coordinate systems are shown as $x=x^{p} \cos (\gamma+\beta(z))-$ $y^{p} \sin (\gamma+\beta(z))$ and $y=x^{p} \sin (\gamma+\beta(z))+y^{p} \cos (\gamma+\beta(z)), z=$ $z^{p}$. $\beta_{0}$ is denoted as the pretwist at the beam tip; then, $\beta(z)=$ $\beta_{0} z / L$ is the pretwist angle of a current beam cross section. The local coordinate system $(s, t, n)$ is defined on the cross section of the beam to describe the geometric configuration and the cross section, where $s$ and $n$ are the circumferential and thickness coordinate variables in Figure 1(c); the notion $(X, Y, Z)$ represents the points off the middle surface; it is different from the notion $(x, y, z)$; the relationship is $X=$ $x+n(d y / d s)$ and $Y=y-n(d x / d s)$. Assume that $(u, v, w)$ and $\left(u_{0}, v_{0}, w_{0}\right)$ represent the displacements of an arbitrary point and a point in the middle surface of the rotating blades on the $x, y$, and $z$ directions, respectively. $\theta_{x}$ and $\theta_{y}$ represent the rotations about the $x$ - and $y$-axis, respectively.

Based on the isotropic constitutive law, the nonlinear partial differential governing equations of motion for the pretwist, presetting, thin-walled rotating cantilever beam were derived by using Hamilton's principle in [11]. Then, Galerkin procedure was applied to obtain the dimensionless governing differential equations of nonlinear vibration for the rotating blade by Yao et al. as follows:

$$
\begin{aligned}
& \ddot{p}(t)+\beta_{12} \dot{p}(t)+\beta_{13} \dot{q}(t)+\omega_{1}^{2} p(t)+\beta_{11} q(t) \\
& -2 \beta_{14} p(t) \Omega_{0} f \cos \Omega_{1} t-\beta_{14} p(t) f^{2} \cos ^{2} \Omega_{1} t \\
& +\beta_{5} p(t) q^{2}(t)+\beta_{5} p^{3}(t)=\beta_{16} \Omega_{1} f \sin \Omega_{1} t, \\
& \ddot{q}(t)+\beta_{22} \dot{p}(t)+\beta_{23} \dot{q}(t)+\omega_{2}^{2} q(t)+\beta_{21} p(t) \\
& \quad-2 \beta_{24} q(t) \Omega_{0} f \cos \Omega_{1} t-\beta_{24} q(t) f^{2} \cos ^{2} \Omega_{1} t \\
& +\beta_{5} p^{2}(t) q(t)+\beta_{5} q^{3}(t)=0,
\end{aligned}
$$

where $p(t)$ and $q(t)$ are the amplitudes of normal modes, $\omega_{1}$ and $\omega_{2}$ are normal frequencies, and $\beta_{12}, \beta_{13}, \beta_{22}$, and $\beta_{23}$ are damping parameters. $\beta_{5}$ plays the role of the nonlinearity, $f$ is the amplitude of excitation, and all the expressions of the coefficients can be found in [11].

We study the case of $2: 1$ internal resonance and primary resonance; the resonant relations are represented as $\omega_{1}^{2}=\Omega_{1}^{2}+$ $\varepsilon \sigma_{1}$ and $\omega_{2}^{2}=(1 / 4) \Omega_{2}^{2}+\varepsilon \sigma_{1}, \Omega_{1}=1$, where $0<\varepsilon \ll 1$ and $\sigma_{1}$ and $\sigma_{2}$ are two detuning parameters.

Using the method of multiple scales, the averaged equations were obtained as follows [11]:

$$
\begin{aligned}
\dot{x}_{1}= & -\mu_{1} x_{1}+\left(-\frac{\sigma_{1}}{2 \Omega_{1}}+\frac{f^{2}}{8 \Omega_{1}} \beta_{14}\right) x_{2} \\
& -\frac{3 \beta_{5}}{8 \Omega_{1}} x_{2}\left(x_{1}^{2}+x_{2}^{2}\right)+\frac{\beta_{5}}{4 \Omega_{1}} x_{2}\left(x_{3}^{2}+x_{4}^{2}\right) \\
& -\frac{1}{2} \beta_{16} f
\end{aligned}
$$




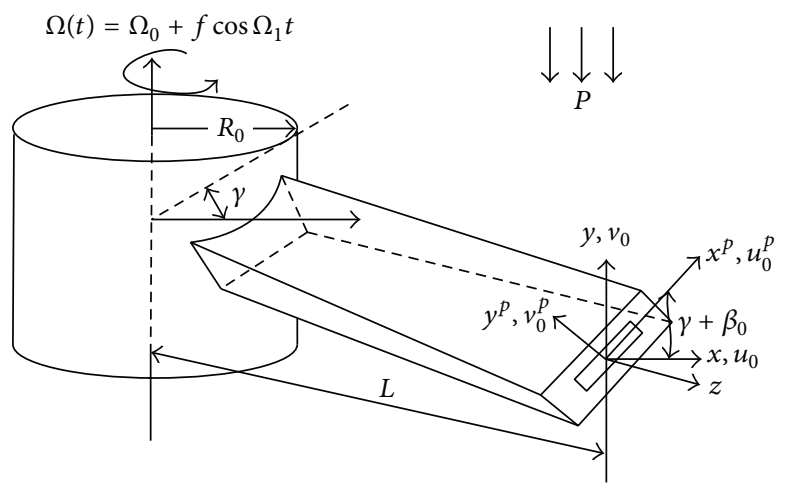

(a)

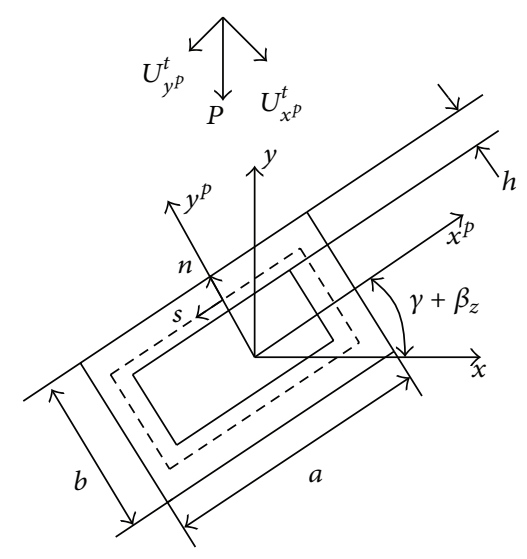

(b)

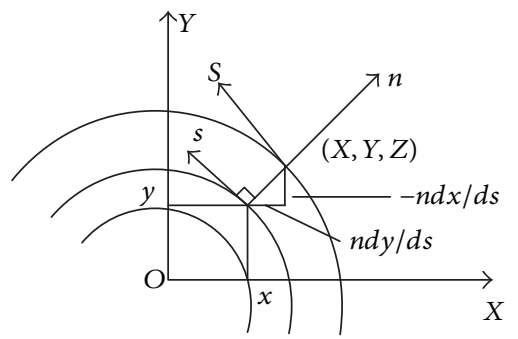

(c)

FIGURE 1: The rotating cantilever beam mode: (a) general view with the global coordinate system, (b) cross section of the rotating beam, and (c) local coordinate systems of the cross section.

$$
\begin{aligned}
\dot{x}_{2}= & \left(\frac{\sigma_{1}}{2 \Omega_{1}}-\frac{3 f^{2}}{8 \Omega_{1}} \beta_{14}\right) x_{1}-\mu_{1} x_{2}+\frac{3 \beta_{5}}{8 \Omega_{1}} x_{1}\left(x_{1}^{2}+x_{2}^{2}\right) \\
& +\frac{\beta_{5}}{4 \Omega_{1}} x_{1}\left(x_{3}^{2}+x_{4}^{2}\right), \\
\dot{x_{3}}= & -\mu_{2} x_{3}+\left(-\frac{\sigma_{2}}{\Omega_{1}}-\frac{\Omega_{0} f}{\Omega_{1}} \beta_{24}+\frac{f^{2}}{2 \Omega_{1}} \beta_{24}\right) x_{4} \\
& -\frac{\beta_{5}}{2 \Omega_{1}} x_{4}\left(x_{1}^{2}+x_{2}^{2}\right)+\frac{3 \beta_{5}}{4 \Omega_{1}} x_{4}\left(x_{3}^{2}+x_{4}^{2}\right),
\end{aligned}
$$$$
\dot{x}_{4}=-\mu_{2} x_{4}+\left(\frac{\sigma_{2}}{\Omega_{1}}-\frac{\Omega_{0} f}{\Omega_{1}} \beta_{24}-\frac{f^{2}}{2 \Omega_{1}} \beta_{24}\right) x_{3}
$$$$
+\frac{\beta_{5}}{2 \Omega_{1}} x_{3}\left(x_{1}^{2}+x_{2}^{2}\right)+\frac{3 \beta_{5}}{4 \Omega_{1}} x_{3}\left(x_{3}^{2}+x_{4}^{2}\right) \text {. }
$$

Equations (2) have a zero solution $\left(x_{1}, x_{2}, x_{3}, x_{4}\right)=$ $(0,0,0,0)$. Without the perturbation parameter $\beta_{16}$, the Jacobian matrix at the origin is

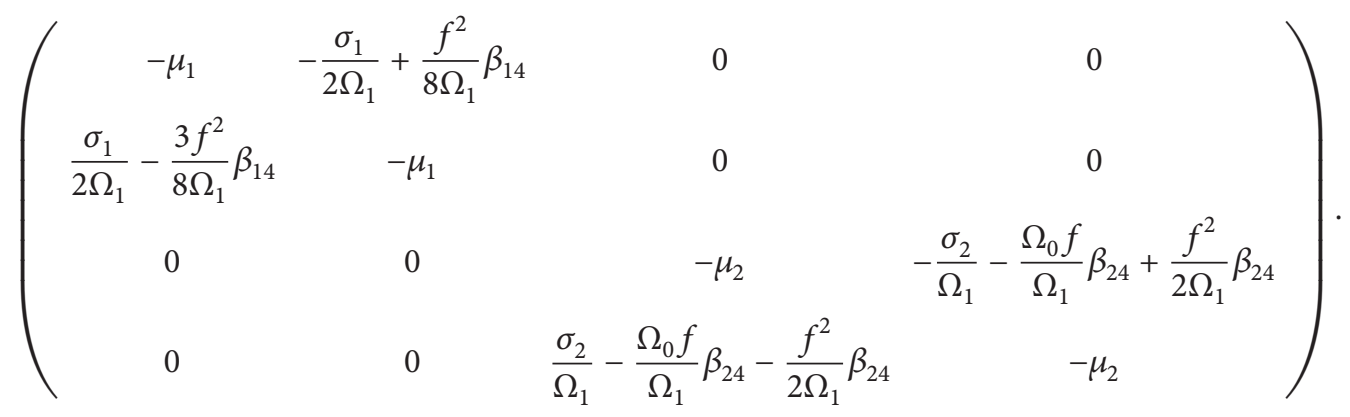


The characteristic equation corresponding to the zero solution is

$$
\begin{aligned}
& P(\lambda)=\left[\lambda^{2}+2 \mu_{1} \lambda+\mu_{1}^{2}-\left(-\frac{\sigma_{1}}{2 \Omega_{1}}+\frac{f^{2}}{8 \Omega_{1}} \beta_{14}\right)\right. \\
& \left.\cdot\left(\frac{\sigma_{1}}{2 \Omega_{1}}-\frac{3 f^{2}}{8 \Omega_{1}} \beta_{14}\right)\right], \\
& {\left[\lambda^{2}+2 \mu_{2} \lambda+\mu_{2}^{2}-\left(-\frac{\sigma_{2}}{\Omega_{1}}-\frac{f \Omega_{0}}{\Omega_{1}} \beta_{24}+\frac{f^{2}}{2 \Omega_{1}} \beta_{24}\right)\right.} \\
& \left.\quad \cdot\left(\frac{\sigma_{2}}{\Omega_{1}}-\frac{f \Omega_{0}}{\Omega_{1}} \beta_{24}-\frac{f^{2}}{2 \Omega_{1}} \beta_{24}\right)\right]=\left[\lambda^{2}+2 \mu_{1} \lambda+\mu_{1}^{2}\right. \\
& \left.\quad-\left(\tilde{\sigma}_{1}-\frac{3}{8} f^{2} r_{1}\right)\left(-\widetilde{\sigma}_{1}+\frac{1}{8} f^{2} r_{1}\right)\right]\left[\lambda^{2}+2 \mu_{2} \lambda+\mu_{2}^{2}\right. \\
& \left.\quad+\left(r_{2}^{2} f^{2}-\widetilde{\sigma}_{2}^{2}\right)\right] .
\end{aligned}
$$

For convenience of the following analysis, let $\widetilde{\sigma}_{1}=$ $\sigma_{1} / 2 \Omega_{1}, \tilde{\sigma}_{2}=\sigma_{2} / \Omega_{1}-\left(f^{2} / 2 \Omega_{1}\right) \beta_{24}, r_{1}=\beta_{14} / \Omega_{1}, r_{2}=$ $\left(\Omega_{0} / \Omega_{1}\right) \beta_{24}, r_{3}=\beta_{5} / \Omega_{1}$, and $r_{4}=\beta_{16}$. When $\mu_{1}=0, \mu_{2}=0$, $\tilde{\sigma}_{2}=r_{2} f$, and $\tilde{\sigma}_{1}>(3 / 8) r_{1} f^{2}$ are simultaneously satisfied, the eigenvalues of system (2) without parameter $r_{4}$ have a nonsemisimple double zero and a pair of pure imaginary eigenvalues $\lambda_{1,2}= \pm i \omega$ and $\lambda_{3,4}=0$, where $\omega^{2}=-\tilde{\sigma}_{1}^{2}+$ $(1 / 2) r_{1} f^{2} \widetilde{\sigma}_{1}-(3 / 64) r_{1}^{2} f^{4}$. Assume $f=1, r_{1}=0, r_{2}=-1 / 2$, and $\sigma_{2}^{\prime}=\widetilde{\sigma}_{2}-r_{2} f$, considering $\sigma_{2}^{\prime}, \mu_{1}, \mu_{2}$, and $r_{4}$ as the perturbation parameters; then, (2) without the perturbation parameters becomes

$$
\begin{aligned}
& \dot{x_{1}}=-\widetilde{\sigma}_{1} x_{2}-\frac{3}{8} r_{3} x_{2}\left(x_{1}^{2}+x_{2}^{2}\right)-\frac{1}{4} r_{3} x_{2}\left(x_{3}^{2}+x_{4}^{2}\right), \\
& \dot{x_{2}}=\widetilde{\sigma}_{1} x_{1}+\frac{3}{8} r_{3} x_{1}\left(x_{1}^{2}+x_{2}^{2}\right)+\frac{1}{4} r_{3} x_{1}\left(x_{3}^{2}+x_{4}^{2}\right), \\
& \dot{x_{3}}=x_{4}-\frac{1}{2} r_{3} x_{4}\left(x_{1}^{2}+x_{2}^{2}\right)+\frac{3}{4} r_{3} x_{4}\left(x_{3}^{2}+x_{4}^{2}\right), \\
& \dot{x_{4}}=\frac{1}{2} r_{3} x_{3}\left(x_{1}^{2}+x_{2}^{2}\right)+\frac{3}{4} r_{3} x_{3}\left(x_{3}^{2}+x_{4}^{2}\right) .
\end{aligned}
$$

In this case, we have

$$
\left(\begin{array}{cccc}
0 & -\widetilde{\sigma}_{1} & 0 & 0 \\
\tilde{\sigma}_{1} & 0 & 0 & 0 \\
0 & 0 & 0 & 1 \\
0 & 0 & 0 & 0
\end{array}\right) .
$$

Using the method in [27], a third-order normal form of (5) is obtained as

$$
\begin{aligned}
& \dot{y}_{1}=-\widetilde{\sigma}_{1} y_{2}-\frac{3}{8} r_{3} y_{2}\left(y_{1}^{2}+y_{2}^{2}\right)-\frac{1}{4} r_{3} y_{2} y_{3}^{2}, \\
& \dot{y}_{2}=\widetilde{\sigma}_{1} y_{1}+\frac{3}{8} r_{3} y_{1}\left(y_{1}^{2}+y_{2}^{2}\right)+\frac{1}{4} r_{3} y_{1} y_{3}^{2}, \\
& \dot{y}_{3}=y_{4}, \\
& \dot{y}_{4}=\frac{1}{2} r_{3} y_{3}\left(y_{1}^{2}+y_{2}^{2}\right)+\frac{3}{4} r_{3} y_{3}^{3} .
\end{aligned}
$$

Normal form with perturbation parameters of system (2) is

$$
\begin{aligned}
\dot{y}_{1}= & -\mu_{1} y_{1}-\widetilde{\sigma}_{1} y_{2}-\frac{3}{8} r_{3} y_{2}\left(y_{1}^{2}+y_{2}^{2}\right)-\frac{1}{4} r_{3} y_{2} y_{3}^{2} \\
& -\frac{1}{2} r_{4}, \\
\dot{y}_{2}= & \widetilde{\sigma}_{1} y_{1}-\mu_{1} y_{2}+\frac{3}{8} r_{3} y_{1}\left(y_{1}^{2}+y_{2}^{2}\right)+\frac{1}{4} r_{3} y_{1} y_{3}^{2}, \\
\dot{y}_{3}= & -\mu_{2} y_{3}+\left(1-\sigma_{2}^{\prime}\right) y_{4}, \\
\dot{y}_{4}= & -\mu_{2} y_{4}+\sigma_{2}^{\prime} y_{3}+\frac{1}{2} r_{3} y_{3}\left(y_{1}^{2}+y_{2}^{2}\right)+\frac{3}{4} r_{3} y_{3}^{3} .
\end{aligned}
$$

We need to transform system (8) to a desired form in order to apply the global perturbation method. Let $\mu_{i} \rightarrow \varepsilon \mu_{i}(i=1,2)$ and $r_{4} \rightarrow \varepsilon r_{4}$, and use the transformations

$$
\begin{aligned}
& y_{1}=\sqrt{I} \cos \phi, \\
& y_{2}=\sqrt{I} \sin \phi, \\
& y_{3}=\left(1-\sigma_{2}^{\prime}\right) u, \\
& y_{4}=\mu_{2} u+v .
\end{aligned}
$$

And substituting (9) into the normal form (8) yields

$$
\begin{aligned}
\dot{u} & =v=\frac{\partial H_{0}}{\partial v}+\varepsilon g^{u}, \\
\dot{v} & =u\left(\frac{1}{2} r_{3} I+\sigma_{2}^{\prime}\left(1-\sigma_{2}^{\prime}\right)-\mu_{2}^{2}\right)+\frac{3}{4} r_{3} u^{3}-2 \varepsilon \mu_{2} v \\
& =-\frac{\partial H_{0}}{\partial u}+\varepsilon g^{v}, \\
\dot{I} & =-2 \varepsilon \mu_{1} I-\varepsilon r_{4} \sqrt{I} \cos \phi=\frac{\partial H_{0}}{\partial \phi}+\varepsilon g^{I}, \\
\dot{\phi} & =\widetilde{\sigma}_{1}+\frac{3}{8} r_{3} I+\frac{1}{4} r_{3} u^{2}+\frac{\varepsilon r_{4} \sin \phi}{2 \sqrt{I}}=-\frac{\partial H_{0}}{\partial I}+\varepsilon g^{\phi}, \\
g^{u} & =\frac{\partial H_{1}}{\partial v}, \\
g^{v} & =-\frac{\partial H_{1}}{\partial u}-2 \mu_{2} v, \\
g^{I} & =\frac{\partial H_{1}}{\partial \phi}-2 \mu_{1} I, \\
g^{\phi} & =-\frac{\partial H_{1}}{\partial I},
\end{aligned}
$$

where the Hamiltonian functions $H_{0}$ and $H_{1}$ are of the following form:

$$
\begin{aligned}
H_{0}= & \frac{1}{2} v^{2}-\frac{1}{4} u^{2} r_{3} I+\frac{u^{2}}{2} \bar{\mu}_{2}-\frac{3}{16} r_{4} u^{4}-\widetilde{\sigma}_{1} I \\
& -\frac{3}{16} r_{3} I^{2}, \\
H_{1}= & -\sqrt{I} r_{4} \sin \phi,
\end{aligned}
$$

where $\bar{\mu}_{2}=\mu_{2}^{2}-\sigma_{2}^{\prime}\left(1-\sigma_{2}^{\prime}\right)$. 


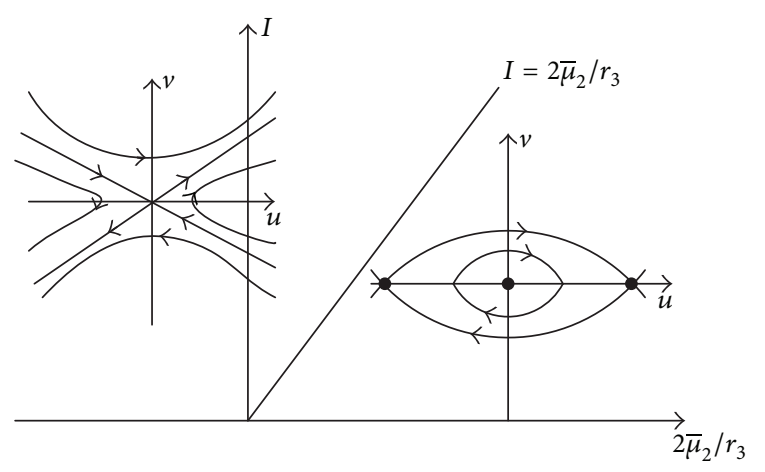

FIgURE 2: Phase portrait of the unperturbed system in the $I-2 \bar{\mu}_{2} / r_{3}$ space.

\section{Dynamics of the Unperturbed System}

Setting $\varepsilon=0$ in system (10), we obtain the unperturbed system. Obviously, the variable $I$ is a constant since $\dot{I}=0$, and the first three equations are completely independent of $\phi$. Thus, we obtain two uncoupled single-degree-of-freedom nonlinear systems:

$$
\begin{aligned}
& \dot{u}=v, \\
& \dot{v}=u\left(\frac{1}{2} r_{3} I-\bar{\mu}_{2}\right)+\frac{3}{4} r_{3} u^{3} .
\end{aligned}
$$

All possible fixed points in $(u, v)$ phase space can be classified as

$$
\begin{aligned}
& P_{1}: u=v=0 ; \\
& P_{2}^{ \pm}: u= \pm \sqrt{\frac{4 \bar{\mu}_{2}-2 r_{3} I}{3 r_{3}}}, \quad v=0,
\end{aligned}
$$

where $\left(4 \bar{\mu}_{2}-2 r_{3} I\right) / 3 r_{3}>0$; that is, $I<2 \bar{\mu}_{2} / r_{3}$ as $r_{3}<0$ or $r_{3}>0$. When $I>2 \bar{\mu}_{2} / r_{3}$, the only solution of system (13) is $P_{1}$, and from the Jacobian matrix evaluated at the trivial solution, $P_{1}$ is a saddle point. At $I=2 \bar{\mu}_{2} / r_{3}$, the trivial solution may bifurcate into three solutions through a pitchfork bifurcation. From the Jacobian matrices evaluated at $P_{1}$ and $P_{2}^{ \pm}$, it is known that $P_{1}$ is a center and $P_{2}^{ \pm}$are two saddle points. The phase portrait is illustrated in Figure 2.

From transformation (9), the variables $I$ and $r$ may actually represent the amplitude and phase of nonlinear oscillations. Therefore, assume that variable $I \geq 0$ and put $I_{1}=0$ and $I_{2}=2 \bar{\mu}_{2} / r_{3}$, such that, for all $I \in\left[I_{1}, I_{2}\right]$, system (13) has two saddle points $P_{2}$ and one center $P_{1}$, which is connected by heteroclinic orbits $\left(u^{h}\left(T_{1}, I\right), v^{h}\left(T_{1}, I\right)\right)$. In fourdimensional space $(u, v, I, \phi)$, the set defined by

$$
\begin{aligned}
M & =\left\{(u, v, I, \phi) \mid u= \pm \sqrt{\frac{4 \bar{\mu}_{2}-2 r_{3} I}{3 r_{3}}}, v=0,0<I\right. \\
& \left.<\frac{2 \bar{\mu}_{2}}{r_{3}}, 0 \leq \phi \leq 2 \pi\right\}
\end{aligned}
$$

is a two-dimensional invariant manifold and it is normally hyperbolic [24].

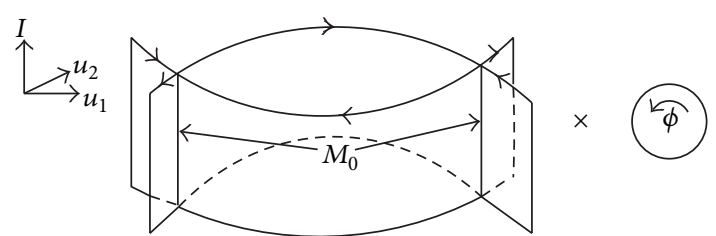

Figure 3: The geometric structures of $M_{0}$ and $\Gamma$.

$M$ has a three-dimensional stable manifold $W^{s}(M)$ and an unstable manifold $W^{u}(M)$. Then, from [24], the existence of the heteroclinic orbits implies that $W^{s}(M)$ and $W^{u}(M)$ intersect nontransversally along a three-dimensional heteroclinic manifold denoted by $\Gamma$, which can be written as

$$
\begin{aligned}
\Gamma & =W^{s}(M) \cap W^{u}(M)=\{(u, v, I, \phi) \mid u \\
& =u^{h}\left(T_{1}, I\right), v=v^{h}\left(T_{1}, I\right), 0<I<\frac{2 \bar{\mu}_{2}}{r_{3}}, \phi \\
& \left.=\int_{0}^{T_{1}} D_{I} H_{0}\left(u^{h}(s, I), v^{h}(s, I), I\right) d s+\phi_{0}\right\},
\end{aligned}
$$

where $\phi_{0}$ is a constant determined by the initial conditions. The geometric structures of the stable and unstable manifolds of $M$ and $\Gamma$ are shown in Figure 3. It is seen that (13) is a Hamilton system with Hamiltonian

$$
H(u, v)=\frac{1}{2} v^{2}-\frac{\eta}{2} u^{2}+\frac{3}{16} r_{3} u^{4}
$$

where $\eta=\bar{\mu}_{2}-(1 / 2) r_{3} I$. Then, we get the expressions of the pair of heteroclinic orbits as follows:

$$
\begin{aligned}
u & = \pm 2 \sqrt{\frac{\eta}{3 r_{3}}} \tanh \left(\frac{\sqrt{2 \eta}}{2} T_{1}\right), \\
v\left(T_{1}\right) & = \pm \sqrt{\frac{2}{3 r_{3}} \eta \operatorname{sech}^{2}\left(\frac{\sqrt{2 \eta}}{2} T_{1}\right) .}
\end{aligned}
$$

The dynamics restricted to the invariant manifold $M$ are described by the following equations:

$$
\begin{aligned}
& \dot{I}=0, \\
& \dot{\phi}=\widetilde{\sigma}_{1}+\frac{3}{8} r_{3} I+\frac{1}{4} r_{3} u^{2},
\end{aligned}
$$

where $I_{1} \leq I \leq I_{2}$. From (19b), we have periodic orbits which are circles for each $I$ when $\widetilde{\sigma}_{1}+(3 / 8) r_{3} I+(1 / 4) r_{3} u^{2} \neq 0$, and the corresponding circle is a circle of fixed points when $\widetilde{\sigma}_{1}+(3 / 8) r_{3} I+(1 / 4) r_{3} u^{2}=0$; that is, $I=I_{r}=\left(-8 \bar{\mu}_{2}-\right.$ $\left.24 \widetilde{\sigma}_{1}\right) / 5 r_{3}$. As homoclinic orbit in $(I, \phi)$ plane is a heteroclinic connection in the four-dimensional $(u, v, I, \phi)$ space which is shown in Figure $4, I_{r}$ is called resonance due to the vanishing frequency of rotation along the $\phi$ direction, and when $I \neq I_{r}$, $\Delta \phi$ is not defined.

Now, we consider the phase shift:

$$
\Delta \phi=\phi\left(+\infty, I_{r}\right)-\phi\left(-\infty, I_{r}\right)
$$




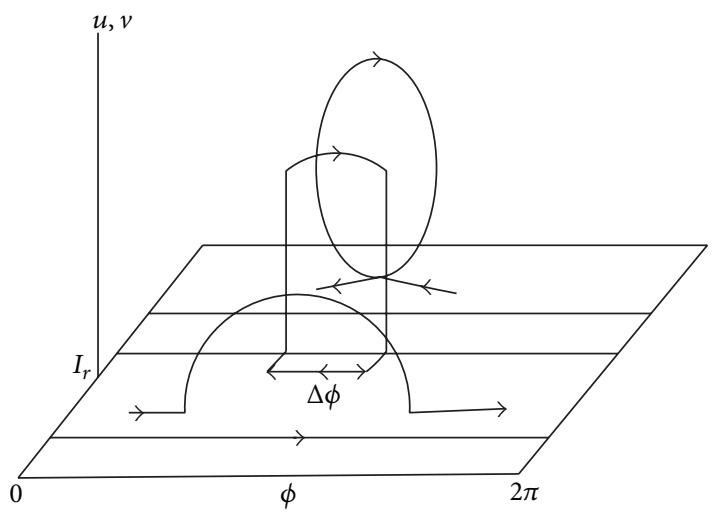

FIGURE 4: The geometry of trajectories homoclinic to the periodic orbits on $M_{0}$ and orbits heteroclinic to fixed points on the resonances.

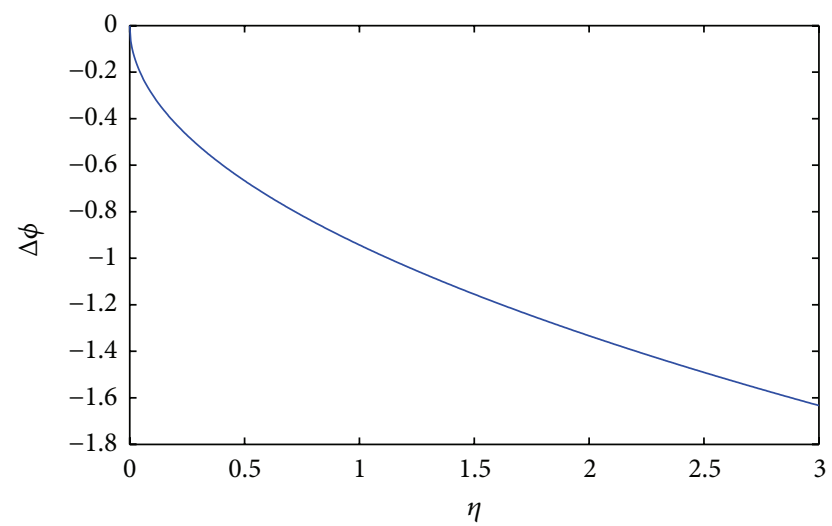

FIgUre 5: The phase shift $\Delta \phi$ defined in (23).

Substituting (18) into (19b) yields

$$
\dot{\phi}=\widetilde{\sigma}_{1}+\frac{3}{8} r_{3} I_{r}+\frac{\eta}{3} \tanh ^{2}\left(\frac{\sqrt{2 h}}{2} T_{1}\right) .
$$

Integrating (21) yields

$$
\begin{aligned}
\phi\left(T_{1}\right)= & \left(\tilde{\sigma}_{1}+\frac{3}{8} r_{3} I_{r}+\frac{\eta}{3}\right) T_{1} \\
& -\frac{\sqrt{2 \eta}}{3} \tanh \left(\frac{\sqrt{2 \eta}}{2} T_{1}\right)+\phi_{0} .
\end{aligned}
$$

Thus, the phase shift is expressed as

$$
\Delta \phi=-\frac{2 \sqrt{2 \eta}}{3} .
$$

$\Delta \phi$ is a function of $\eta$. It is illustrated in Figure 5.

\section{Dynamics of the Perturbed System}

As the manifold $M$ along with its stable manifold $W^{s}(M)$ and unstable manifold $W^{u}(M)$ is invariant under sufficiently small perturbations [24], under perturbation (when $\varepsilon \neq 0$ ),
$M$ becomes a locally invariant two-dimensional manifold $M_{\varepsilon}$ described as follows:

$$
\begin{aligned}
M_{\varepsilon} & =\left\{\left(u_{\varepsilon}, v_{\varepsilon}, I, \phi\right) \mid u_{\varepsilon}(I, \phi)= \pm 2 \sqrt{\frac{\eta}{3 r_{3}}}+\varepsilon u_{1}(I, \phi)\right. \\
& +o\left(\varepsilon^{2}\right), v_{\varepsilon}(I, \phi)=0+\varepsilon v_{1}(I, \phi)+o\left(\varepsilon^{2}\right), 0<I \\
& \left.<\frac{2 \bar{\mu}_{2}}{3 r_{3}}, 0 \leq \phi \leq 2 \pi\right\} .
\end{aligned}
$$

The flow on $M_{\varepsilon}$ is obtained by substituting $\left(u_{\varepsilon}, v_{\varepsilon}\right)$ into (10):

$$
\begin{aligned}
& \dot{I}=-2 \varepsilon \mu_{1} I-\varepsilon r_{4} \sqrt{I} \cos \phi+o\left(\varepsilon^{2}\right), \\
& \dot{\phi}=\widetilde{\sigma}_{1}+\frac{3}{8} r_{3} I+\frac{1}{4} r_{3} u^{2}+\frac{\varepsilon r_{4} \sin \phi}{2 \sqrt{I}}+o\left(\varepsilon^{2}\right) .
\end{aligned}
$$

Introduce the scale transformations

$$
\begin{aligned}
I & =I_{r}+\sqrt{\varepsilon} h, \\
\tau & =\sqrt{\varepsilon} T_{1} .
\end{aligned}
$$

Substituting transformations (26) into (25) yields

$$
\begin{aligned}
h^{\prime}= & -2 \mu_{1} I_{r}-\sqrt{I_{r}} r_{4} \cos \phi-\sqrt{\varepsilon} h\left(2 \mu_{1}+\frac{r_{4}}{2 \sqrt{I}} \cos \phi\right) \\
& +o(\varepsilon), \\
\phi^{\prime}= & \frac{1}{24} r_{3} h+\frac{r_{4} \sin \phi}{2 \sqrt{I}} \sqrt{\varepsilon}+o(\varepsilon) .
\end{aligned}
$$

When $\varepsilon=0$, (27) is reduced to

$$
\begin{aligned}
h^{\prime} & =-2 \mu_{1} I_{r}-\sqrt{I_{r}} r_{4} \cos \phi, \\
\phi^{\prime} & =\frac{1}{24} r_{3} h,
\end{aligned}
$$

which is a Hamilton system with Hamiltonian

$$
H(h, \phi)=-2 \mu_{1} I_{r} \phi-\sqrt{I_{r}} r_{4} \sin \phi+\frac{1}{12} r_{3} h^{2} .
$$

The fixed points of Hamilton system (28) are given by

$$
\begin{aligned}
& p\left(0, \phi_{s}\right)=\left(0, \pi-\arccos \left(\frac{2 \mu_{1} \sqrt{I_{r}}}{r_{4}}\right)\right), \\
& q\left(0, \phi_{c}\right)=\left(0, \pi+\arccos \left(\frac{2 \mu_{1} \sqrt{I_{r}}}{r_{4}}\right)\right) .
\end{aligned}
$$

The Jacobian matrix of (28) evaluated at these fixed points is

$$
J=\left(\begin{array}{cc}
0 & \sqrt{I_{r}} r_{4} \sin \phi_{s, c} \\
\frac{1}{24} r_{3} & 0
\end{array}\right) .
$$

It is easy to find that $p$ is a saddle point and $q$ is a center. Therefore, there exists a homoclinic orbit connecting $p$ to 


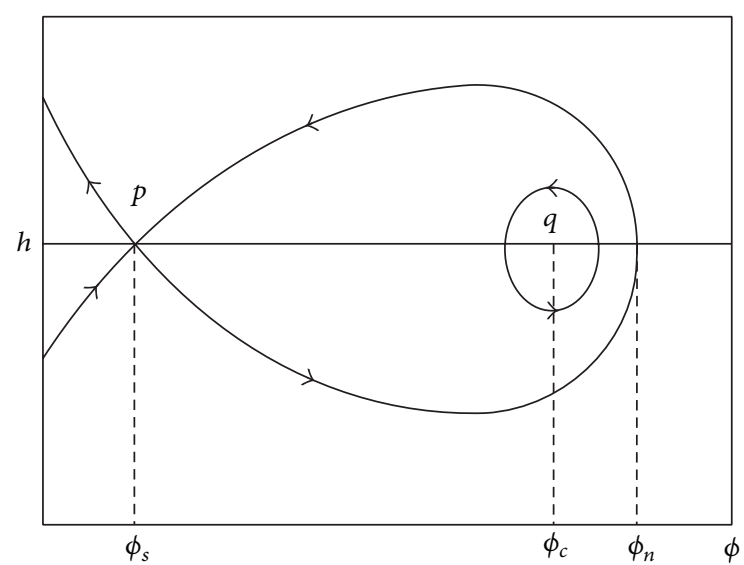

FIGURe 6: Dynamics of unperturbed system (28) on $M_{\varepsilon}$.

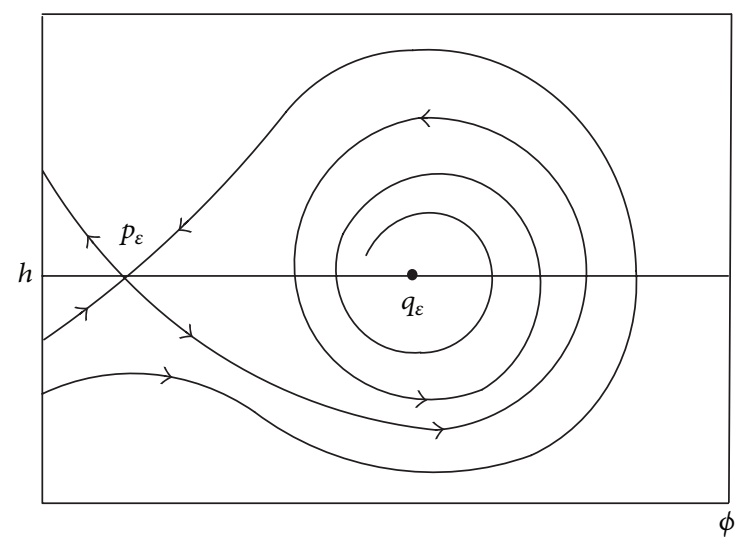

Figure 7: Dynamics of perturbed system (27) on $M_{\varepsilon}$.

itself. The phase portrait of system (28) is shown in Figure 6. By the analysis of Kovacic and Wiggins [24], we can obtain that, for sufficiently small $\varepsilon, p$ remains a saddle point and $q$ becomes a hyperbolic sink $q_{\varepsilon}$.

The phase portrait of perturbed system (27) is given in Figure 7; Hamilton function remains constant on the homoclinic orbit; that is, $\bar{H}\left(0, \phi_{n}\right)=\bar{H}\left(0, \phi_{s}\right)$; then, we have

$$
\begin{aligned}
- & 2 \mu_{1} I_{r} \phi_{n}-\sqrt{I_{r}} r_{4} \sin \phi_{n} \\
= & -2 \mu_{1} I_{r}\left[\pi-\arccos \left(\frac{2 \mu_{1} \sqrt{I_{r}}}{r_{4}}\right)\right] \\
& -\sqrt{I_{r}} r_{4} \sin \left[\pi-\arccos \left(\frac{2 \mu_{1} \sqrt{I_{r}}}{r_{4}}\right)\right] .
\end{aligned}
$$

In order to consider the dynamics on $M_{\varepsilon}$ in the neighborhood of $I=I_{r}$, an annulus $A_{\varepsilon}$ is defined as

$$
\begin{aligned}
A_{\varepsilon} & =\left\{(u, v, h, \phi) \mid u=u\left(I_{r}+\sqrt{\varepsilon} h, \phi\right), v\right. \\
& \left.=v\left(I_{r}+\sqrt{\varepsilon} h, \phi\right),|h|<h_{0}, 0 \leq \phi \leq 2 \pi\right\},
\end{aligned}
$$

where $h_{0}$ is a constant, which is chosen sufficient large so that the unperturbed homoclinic orbits are enclosed within the annulus. Denote $W^{s}\left(A_{\varepsilon}\right)$ and $W^{u}\left(A_{\varepsilon}\right)$ as the threedimensional stable and unstable manifolds of $A_{\varepsilon}$, which are subsets of $W^{s}\left(A_{\varepsilon}\right)$ and $W^{u}\left(A_{\varepsilon}\right)$, respectively. According to the analysis of [24], the existence of an orbit homoclinic to a saddle-focus point $q_{\varepsilon}$ can lead to chaos. This type of homoclinic orbit is called Shilnikov-type homoclinic orbit. The point $q_{\varepsilon}$ on $A_{\varepsilon}$ has an orbit that comes out of $A_{\varepsilon}$ in the four-dimensional space and may return to the annulus; it may approach $q_{\varepsilon}$ asymptotically as $t \rightarrow \infty$ and eventually complete a Shilnikov-type homoclinic orbit as shown in Figure 8.

We need to confirm the existence of a Shilnikov type homoclinic orbit in two steps. First, we show $W^{u}\left(A_{\varepsilon}\right) \subset$ $W^{s}\left(A_{\varepsilon}\right)$ by using Melnikov theory when Melnikov function has a simple zero. Second, we determine whether or not the trajectory in $W^{u}\left(A_{\varepsilon}\right)$ comes back in the domain of attraction of $q_{\varepsilon}$. Based on [24], the higher dimensional Melnikov function is given as

$$
M^{I_{r}}=\int_{-\infty}^{+\infty}\left(\frac{\partial H_{0}}{\partial u} g^{u}+\frac{\partial H_{0}}{\partial v} g^{v}+\frac{\partial H_{0}}{\partial I} g^{I}\right) d T_{1} .
$$

Using the division of integral method and the abovementioned analysis, (34) can be expressed as

$$
\begin{aligned}
M^{I_{r}} & =\int_{-\infty}^{+\infty}\left(-\frac{d H_{1}}{d T_{1}}-2 \mu_{2} \dot{u} v+2 \mu_{1} I_{r} \dot{\phi}\right) d T_{1} \\
& =M_{1}+M_{2}+M_{3} .
\end{aligned}
$$

With the aforementioned analysis, the first term can be evaluated as

$$
\begin{aligned}
M_{1} & =-\int_{-\infty}^{+\infty} \frac{d H_{1}}{d T_{1}} d T_{1}=\sqrt{I} r_{4}[\sin \phi(+\infty) \\
& -\sin \phi(-\infty)]=\sqrt{I} r_{4}[\cos \phi(-\infty) \sin \Delta \phi \\
& -\sin \phi(-\infty)(1-\cos \Delta \phi)] \\
& =\sqrt{I} r_{4}\left[-\frac{2 \mu_{1} \sqrt{I}}{r_{4}} \sin \Delta \phi\right. \\
& \left.+\sqrt{1-\frac{2 \mu_{1}^{2} I_{r}}{r_{4}^{2}}}(\cos \Delta \phi-1)\right] .
\end{aligned}
$$

The second term can be simplified as

$$
\begin{aligned}
M_{2} & =2 \mu_{2} \int_{-\infty}^{+\infty} \frac{2}{3 r_{3}} \eta^{2} \operatorname{sech}^{4}\left(\frac{\sqrt{2 \eta}}{2} T_{1}\right) d T_{1} \\
& =-\left.\frac{16 \sqrt{2} \mu_{2} \eta^{3 / 2}}{9 r_{3}}\right|_{I=I_{r}} .
\end{aligned}
$$

The third term is changed into

$$
M_{3}=\int_{-\infty}^{+\infty} 2 \mu_{1} I_{r} \dot{\phi} d T_{1}=2 \mu_{1} I_{r} \Delta \phi
$$




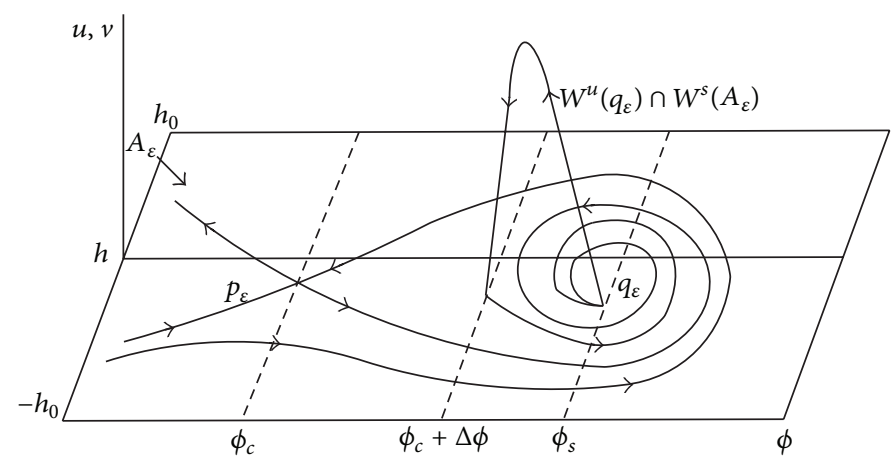

FIGURE 8: Shilnikov-type homoclinic orbit to $p_{\varepsilon}$.

By (36), (37), and (38), the Melnikov function may be expressed as

$$
\begin{aligned}
& M^{I_{r}}=r_{4} \sqrt{I}\left[-\frac{2 \mu_{1} \sqrt{I}}{r_{4}} \sin \Delta \phi\right. \\
& \left.+(\cos \Delta \phi-1) \sqrt{1-\frac{4 \mu_{1}^{2} I_{r}}{r_{4}^{2}}}\right]-\frac{16 \sqrt{2} \mu_{2} \eta^{3 / 2}}{9 r_{3}} \\
& +2 \mu_{1} I_{r} \Delta \phi .
\end{aligned}
$$

Now, we can require that the Melnikov function has a simple zero. That is, we require

$$
\begin{aligned}
& r_{4} \sqrt{I}\left[-\frac{2 \mu_{1} \sqrt{I}}{r_{4}} \sin \Delta \phi+(\cos \Delta \phi-1) \sqrt{1-\frac{4 \mu_{1}^{2} I_{r}}{r_{4}}}\right] \\
& -\frac{16 \sqrt{2} \mu_{2}}{9 r_{3}} \eta^{3 / 2}+2 \mu_{1} I_{r} \Delta \phi=0 .
\end{aligned}
$$

Next, we examine whether the orbit on $W^{u}\left(q_{\varepsilon}\right)$ returns to the domain of attraction of $q_{\varepsilon}$. The condition is given by

$$
\phi_{s}<\phi_{c}+\Delta \phi+2 m \pi<\phi_{n}
$$

where $m$ is an integer, $\phi_{s}, \phi_{c}$, and $\phi_{n}$ are given by (30) and (31), and $\Delta \phi$ is the change of angle. According to [24], when conditions (40) and (41) are satisfied simultaneously, there exists the Shilnikov-type chaos in the sense of Smale horseshoes in system (2).

\section{Numerical Simulation of Chaotic Motions}

Now fixed parameters are used in the abovementioned theory to simplify the calculation. Letting

$$
\begin{aligned}
& \mu_{1}=\mu_{2}=\mu, \\
& \beta=\frac{2 r_{4}}{\mu}, \\
& I_{r}=1,
\end{aligned}
$$

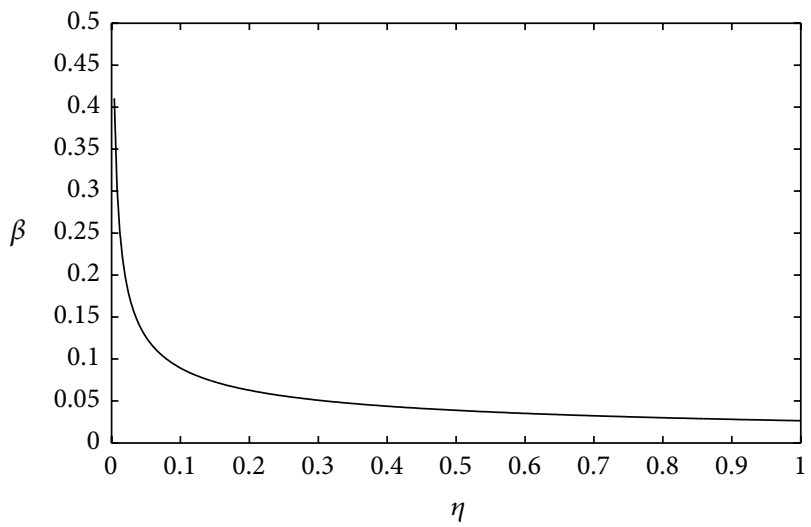

Figure 9: The zeros of Melnikov's function.

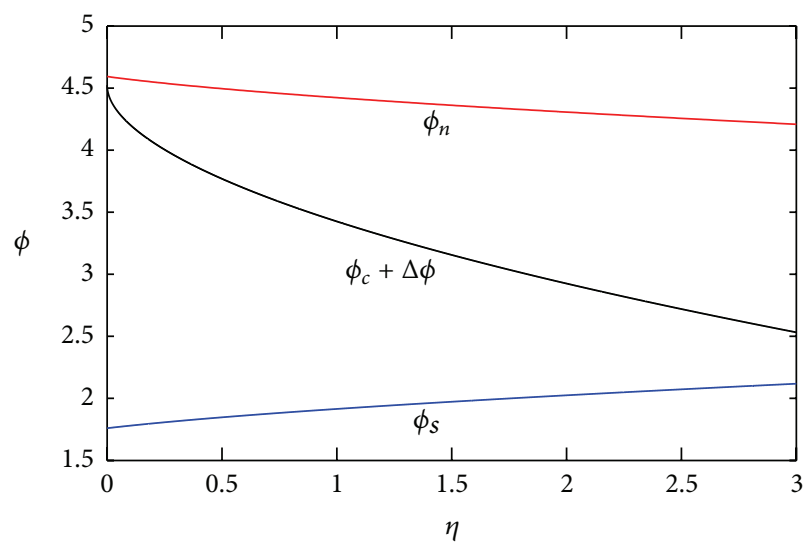

Figure 10: Graphs of $\phi_{s}, \phi_{c}+\Delta \phi$, and $\phi_{n}$.

condition (41) becomes

$$
=\frac{1-\cos \Delta \phi}{\sqrt{(\cos \Delta \phi-1)^{2}+\left((-36.28 \sqrt{2} / 9) \eta^{3 / 2}-\Delta \phi+\sin \Delta \phi\right)^{2}}} .
$$

From (23), $\Delta \phi$ is a function of $\eta$; then, $\beta$ is a function of $\eta$. The figure of $\beta$ shows that $\beta$ exists when $\eta \in(0,1)$, so Melnikov function $M^{I_{r}}(\beta, \eta)$ has a simple zero (Figure 9). $\phi_{s}, \phi_{c}$, and $\phi_{n}$ are presented in Figure 10; we can see $\phi_{s}<\phi_{c}+\Delta \phi<\phi_{n}$; 


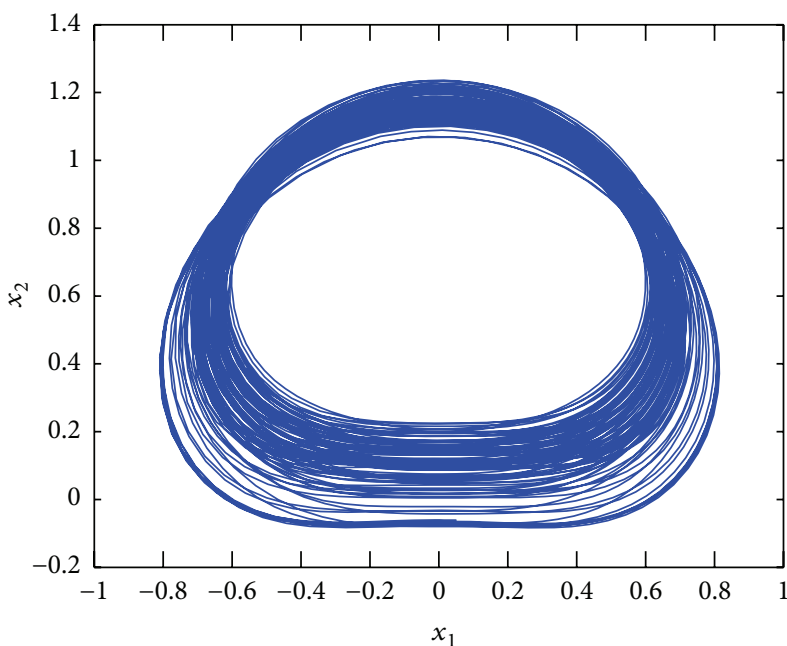

(a)

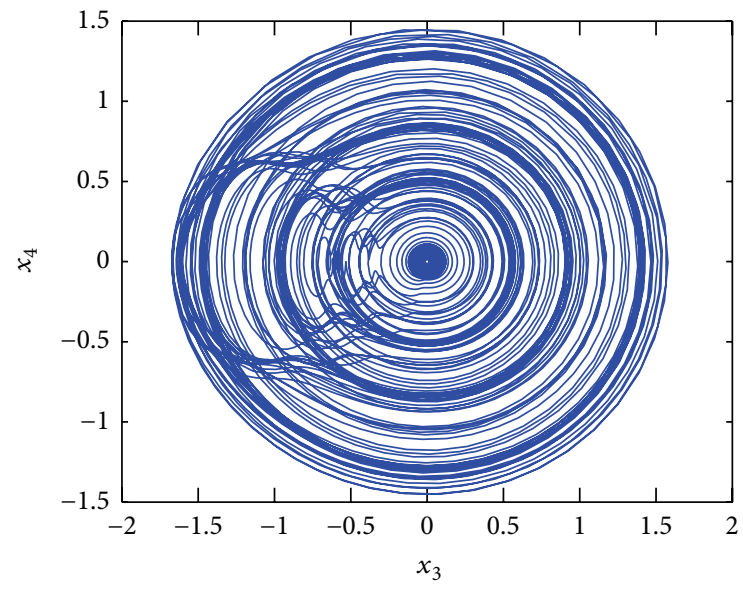

(c)

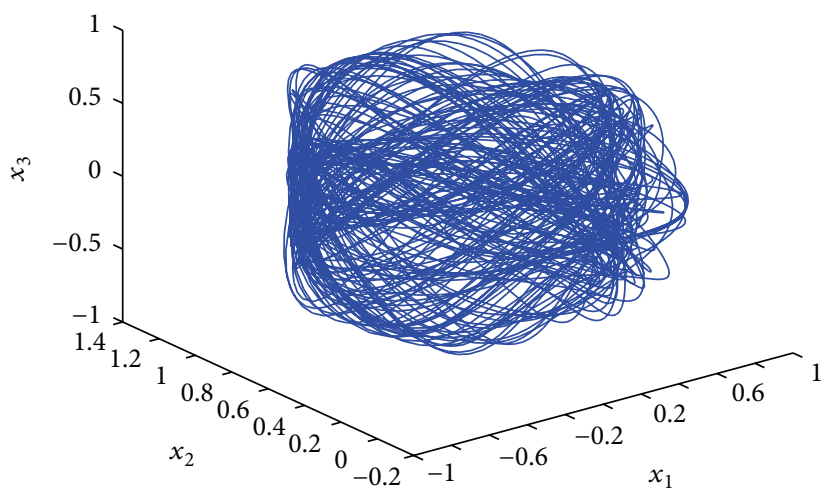

(e)

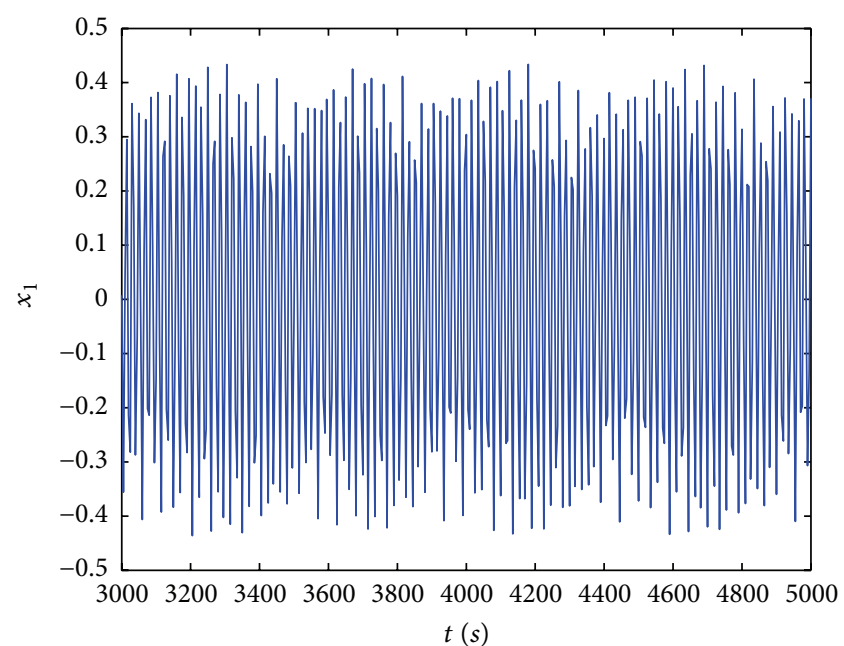

(b)

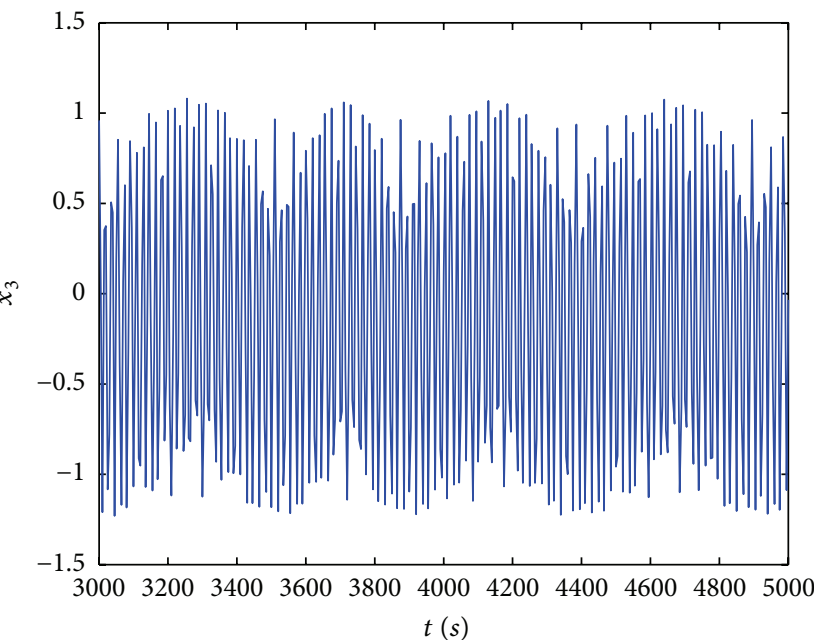

(d)

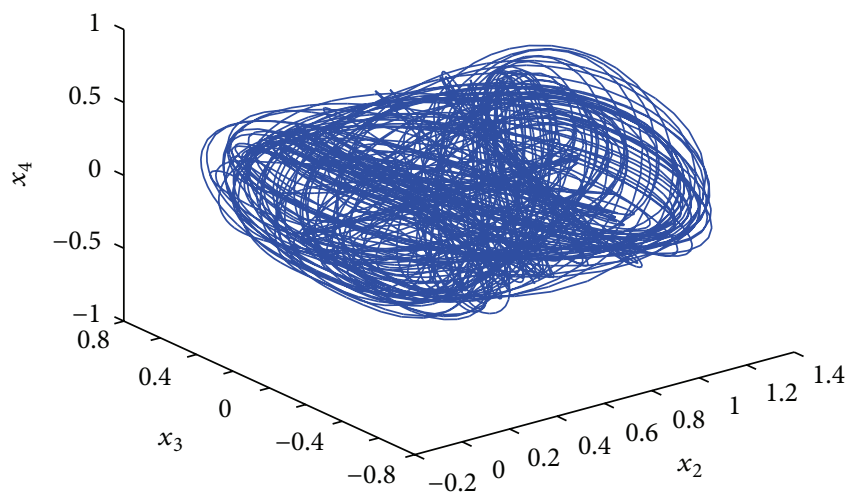

(f)

FIGURE 11: The chaotic motions of the compressor blade based on (2): (a) the phase portrait on plane $\left(x_{1}, x_{2}\right),(\mathrm{b})$ the waveform on plane $\left(t, x_{1}\right)$, (c) the phase portrait on plane $\left(x_{3}, x_{4}\right),(\mathrm{d})$ the waveform on plane $\left(t, x_{3}\right)$, (e) the phase portrait in three-dimensional space $\left(x_{1}, x_{2}, x_{3}\right)$, and (f) the phase portrait in three-dimensional space $\left(x_{2}, x_{3}, x_{4}\right)$.

that is, condition (41) is satisfied. Then, $q_{\varepsilon}$ has a Shilnikov homoclinic orbit for sufficiently small $\varepsilon$. We choose (1) and (2) to do numerical simulations. We use numerical approach to explore the existence of chaotic motions of the rotating thin-walled blade. In Figure 10, we show the existence of the chaotic responses of the thin-walled blade to the forcing excitation. $\beta_{16}=8.8$, and other parameters and initial conditions were chosen as $\mu=0.001, \sigma_{1}=12, \beta_{14}=0, \beta_{5}=-17.64$, $\mu_{2}=0.001, \sigma_{2}=11 / 40, \Omega_{0}=5, \beta_{24}=-4, x_{10}=-0.052$, $x_{20}=0.061, x_{30}=0.042$, and $x_{40}=-0.051$. Figure 11 


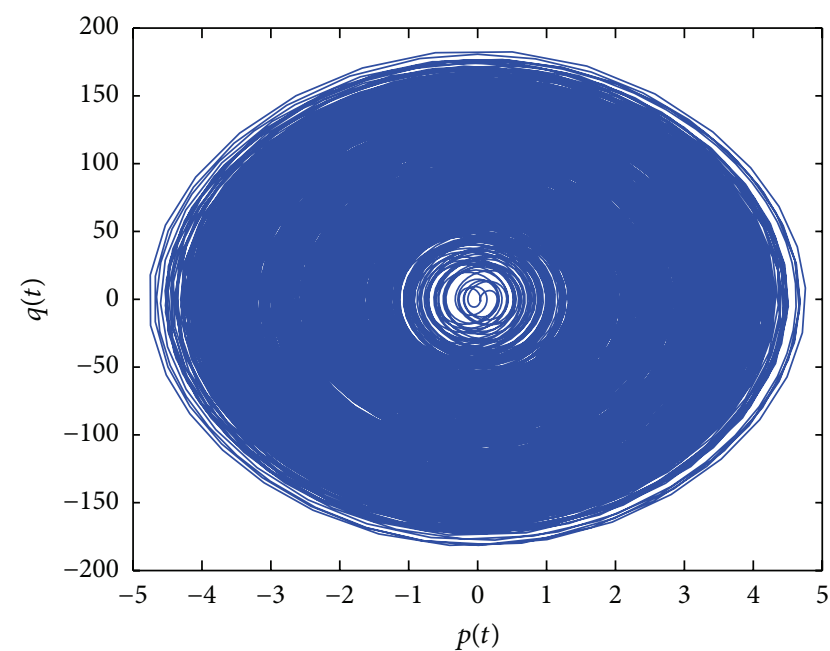

(a)

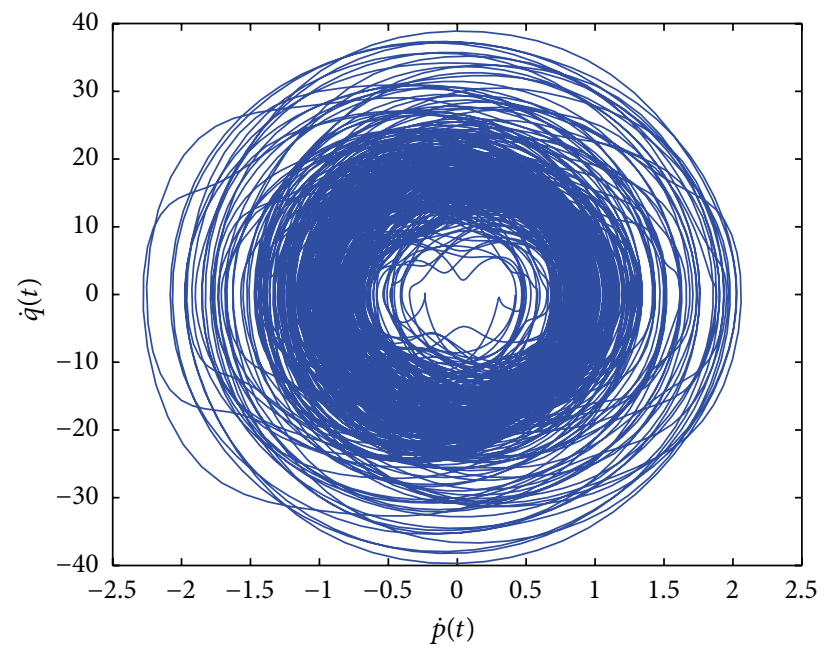

(c)

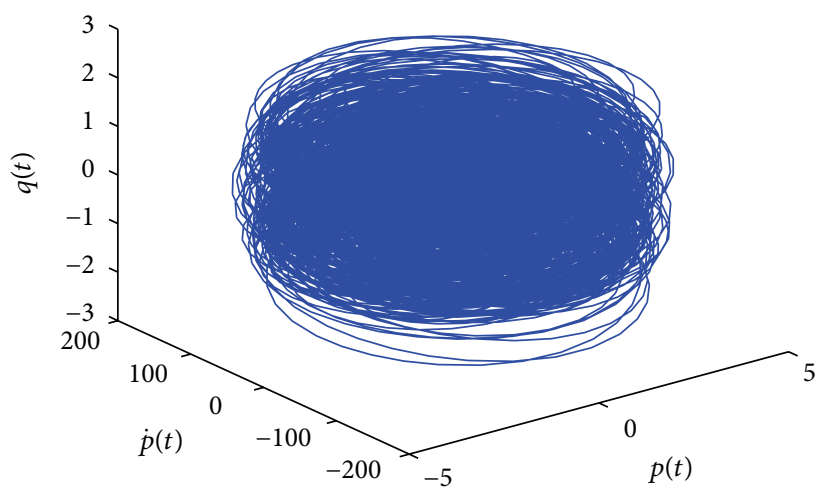

(e)

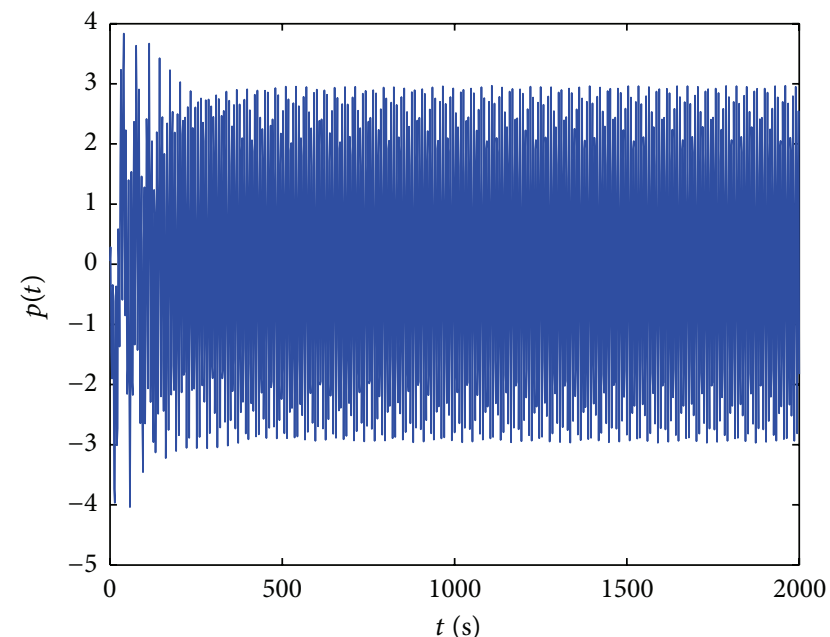

(b)

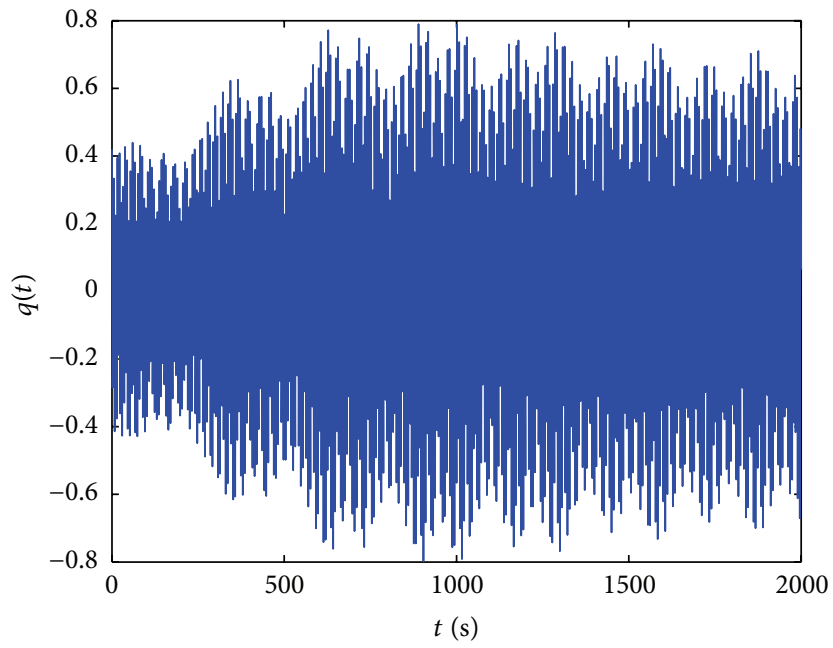

(d)

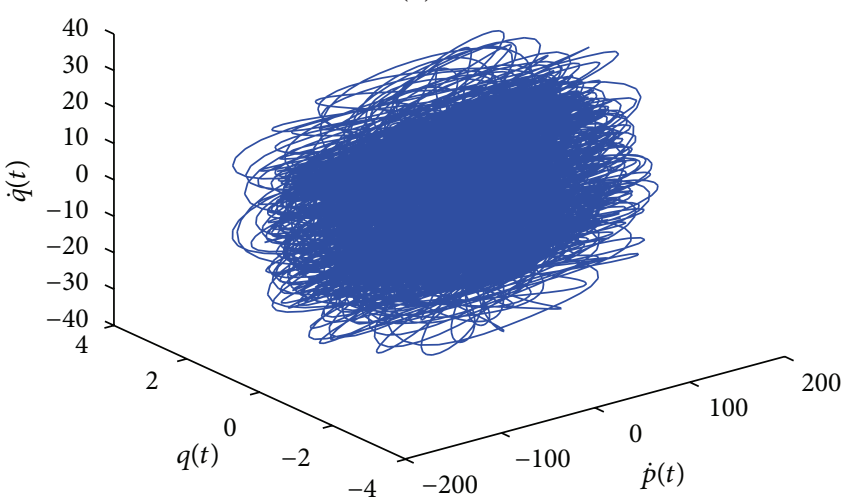

(f)

FIGURE 12: The chaotic motions of the compressor blade based on (1): (a) the phase portrait on plane $\left(x_{1}, x_{2}\right)$, (b) the waveform on plane $\left(t, x_{1}\right)$, (c) the phase portrait on plane $\left(x_{3}, x_{4}\right),(\mathrm{d})$ the waveform on plane $\left(t, x_{3}\right)$, (e) the phase portrait in three-dimensional space $\left(x_{1}, x_{2}, x_{3}\right)$, and (f) the phase portrait in three-dimensional space $\left(x_{2}, x_{3}, x_{4}\right)$.

shows the phase portraits on the planes $\left(x_{1}, x_{2}\right),\left(x_{3}, x_{4}\right)$, $\left(x_{1}, x_{2}, x_{3}\right),\left(x_{2}, x_{3}, x_{4}\right)$ and the wave forms on plane $\left(t, x_{1}\right)$, $\left(t, x_{3}\right)$ based on (2). With the same parameters, we get the portraits on the planes $\left(x_{1}, x_{2}\right),\left(x_{3}, x_{4}\right),\left(x_{1}, x_{2}, x_{3}\right),\left(x_{2}\right.$, $\left.x_{3}, x_{4}\right)$ and the wave forms on plane $\left(t, x_{1}\right),\left(t, x_{3}\right)$ based on (1). They are shown in Figure 12; the chaotic motion 
demonstrated in Figures 11 and 12 is Shilnikov-type multipulse chaotic motion. Therefore, the numerical results agree with the theoretical predictions qualitatively.

\section{Conclusions}

The global bifurcations and chaotic dynamics of the thinwalled compressor blade with varying speed are investigated for the first time by using the analytical and numerical approaches simultaneously when the averaged equations have one nonsemisimple double zero and a pair of pure imaginary eigenvalues. The study is focused on coexistence of 2:1 internal resonance and primary resonance. Normal theory is utilized to find the explicit expressions of the simpler normal form of the averaged equations with a double zero and a pair of pure imaginary eigenvalues. Based on the Melnikov method and its extensions to resonance cases developed by Kovacic and Wiggins, the thin-walled compressor blade can undergo homoclinic bifurcation and the Shilnikov-type homoclinic orbit; that is, there exists chaotic motion in full four-dimensional averaged system. Finally, the Dynamics software is used to perform numerical simulation. The numerical results show the existence of chaotic motions in the averaged equations, which illustrate the predictions obtained by the theoretical analysis. The chaotic motions in averaged equations can lead to the amplitude modulated chaotic oscillations in the original system under certain conditions. Therefore, there are Shilnikov-type single-pulse chaotic motions for the thin-walled rotating compressor blade. This is the extension of the results obtained by Yao et al. [11]. We believe that our results give a direct explanation for the jumping behaviors observed in this class of the compressor blade under in-plane and moment excitations.

\section{Competing Interests}

The authors declare that they have no competing interests.

\section{Acknowledgments}

This work is supported by the National Natural Science Foundation of China (11572148).

\section{References}

[1] S. M. Yang and S. M. Tsao, "Dynamics of a pretwisted blade under nonconstant rotating speed," Computers \& Structures, vol. 62, no. 4, pp. 643-651, 1997.

[2] G. Surace, V. Anghel, and C. Mares, "Coupled bendingbending-torsion vibration analysis of rotating pretwisted blades: an integral formulation and numerical examples," Journal of Sound and Vibration, vol. 206, no. 4, pp. 473-486, 1997.

[3] G. Şakar and M. Sabuncu, "Dynamic stability of a rotating asymmetric cross-section blade subjected to an axial periodic force," International Journal of Mechanical Sciences, vol. 45, no. 9, pp. 1467-1482, 2003.

[4] B. O. Al-Bedoor and A. A. Al-Qaisia, "Stability analysis of rotating blade bending vibration due to torsional excitation," Journal of Sound and Vibration, vol. 282, no. 3-5, pp. 1065-1083, 2005.
[5] D. M. Tang and E. H. Dowell, "Nonlinear response of a nonrotating rotor blade to a periodic gust," Journal of Fluids and Structures, vol. 10, no. 7, pp. 721-742, 1996.

[6] S.-T. Choi and Y.-T. Chou, "Vibration analysis of elastically supported turbomachinery blades by the modified differential quadrature method," Journal of Sound and Vibration, vol. 240, no. 5, pp. 937-953, 2001.

[7] D. Poirel and S. J. Price, "Bifurcation characteristics of a twodimensional structurally non-linear airfoil in turbulent flow," Nonlinear Dynamics, vol. 48, no. 4, pp. 423-435, 2007.

[8] W. Lacarbonara, H. Arvin, and F. Bakhtiari-Nejad, "A geometrically exact approach to the overall dynamics of elastic rotating blades-part 1: linear modal properties," Nonlinear Dynamics, vol. 70, no. 1, pp. 659-675, 2012.

[9] H. Arvin, W. Lacarbonara, and F. B. Nejad, "A geometrically exact approach to the overall dynamics of elastic rotating blades part II: flapping nonlinear normal modes," Nonlinear Dynamics, vol. 70, no. 3, pp. 2279-2301, 2012.

[10] M. H. Yao, Y. P. Chen, and W. Zhang, "Nonlinear vibrations of blade with varying rotating speed," Nonlinear Dynamics, vol. 68, no. 4, pp. 487-504, 2012.

[11] M. H. Yao, W. Zhang, and Y. P. Chen, "Analysis on nonlinear oscillations and resonant responses of a compressor blade," Acta Mechanica, vol. 225, no. 12, pp. 3483-3510, 2014.

[12] F. Wang and W. Zhang, "Stability analysis of a nonlinear rotating blade with torsional vibrations," Journal of Sound and Vibration, vol. 331, no. 26, pp. 5755-5773, 2012.

[13] S.-Y. Oh, O. Song, and L. Librescu, "Effects of pretwist and presetting on coupled bending vibrations of rotating thinwalled composite beams," International Journal of Solids \& Structures, vol. 40, no. 5, pp. 1203-1224, 2003.

[14] F. S. M. Jarrar and M. N. Hamdan, "Nonlinear vibrations and buckling of a flexible rotating beam: a prescribed torque approach," Mechanism \& Machine Theory, vol. 42, no. 8, pp. 919939, 2007.

[15] A. Warminska, E. Manoach, J. Warminski, and S. Samborski, "Regular and chaotic oscillations of a Timoshenko beam subjected to mechanical and thermal loadings," Continuum Mechanics and Thermodynamics, vol. 27, no. 4-5, pp. 719-737, 2015.

[16] Z. C. Feng and P. R. Sethna, "Global bifurcations in the motion of parametrically excited thin plates," Nonlinear Dynamics, vol. 4, no. 4, pp. 389-408, 1993.

[17] W. Zhang and M. H. Yao, "Theories of multi-pulse global bifurcations for high-dimensional systems and application to cantilever beam," International Journal of Modern Physics B, vol. 22, no. 24, pp. 4089-4141, 2008.

[18] W. Q. Yu and F. Q. Chen, "Global bifurcations and chaos in externally excited cyclic systems," Communications in Nonlinear Science \& Numerical Simulation, vol. 15, no. 12, pp. 4007-4019, 2010.

[19] W. Zhang, M. H. Yao, and J. H. Zhang, "Using the extended Melnikov method to study the multi-pulse global bifurcations and chaos of a cantilever beam," Journal of Sound and Vibration, vol. 319, no. 1-2, pp. 541-569, 2009.

[20] M. H. Yao, W. Zhang, and J. W. Zu, "Multi-pulse chaotic dynamics in non-planar motion of parametrically excited viscoelastic moving belt," Journal of Sound and Vibration, vol. 331, no. 11, pp. 2624-2653, 2012.

[21] M. H. Yao and W. Zhang, "Multi-pulse chaotic motions of high-dimension nonlinear system for a laminated composite 
piezoelectric rectangular plate," Meccanica, vol. 49, no. 2, pp. 365-392, 2014.

[22] M. H. Yao, W. Zhang, and D. M. Wang, "Modeling and chaotic dynamics of the laminated composite piezoelectric rectangular plate," Mathematical Problems in Engineering, vol. 2014, Article ID 345072, 19 pages, 2014.

[23] S. Wiggins, Global Bifurcations and Chaos-Analytial Methods, vol. 73 of Applied Mathematical Sciences, Springer, 1988.

[24] G. Kovacic and S. Wiggins, "Orbits homoclinic to resonances, with an application to chaos in a model of the forced and damped sine-Gordon equation," Physica D: Nonlinear Phenomena, vol. 57, no. 1-2, pp. 185-225, 1992.

[25] G. Haller and S. Wiggins, "N-pulse homoclinic orbits in perturbations of resonant hamiltonian systems," Archive for Rational Mechanics \& Analysis, vol. 130, no. 1, pp. 25-101, 1995.

[26] G. Haller, Chaos Near Resonance, vol. 138 of Applied Mathematical Sciences, Springer, New York, NY, USA, 1999.

[27] W. Zhang, F. X. Wang, and J. W. Zu, "Computation of normal forms for high dimensional non-linear systems and application to non-planar non-linear oscillations of a cantilever beam," Journal of Sound and Vibration, vol. 278, no. 4-5, pp. 949-974, 2004. 


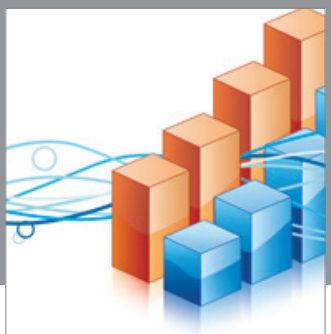

Advances in

Operations Research

vatem alat4

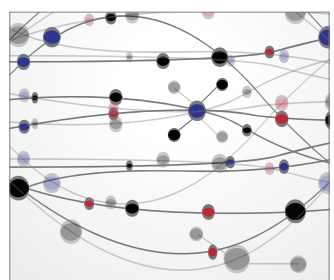

\section{The Scientific} World Journal
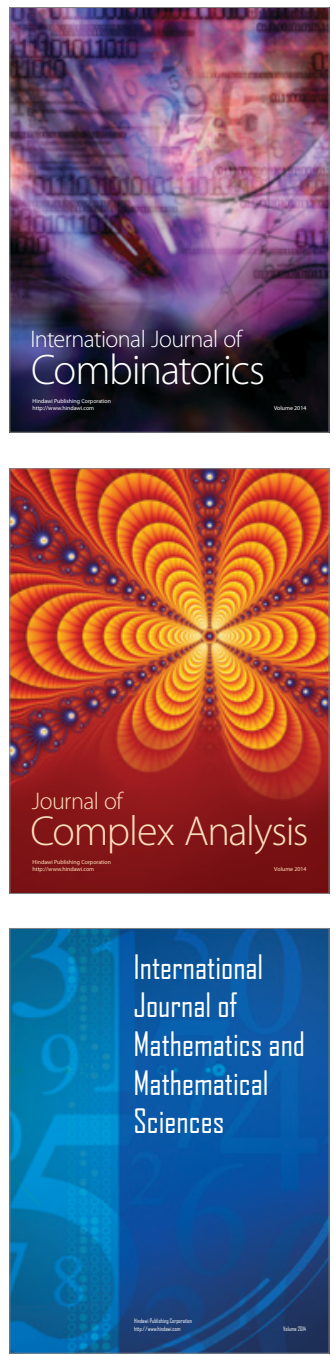
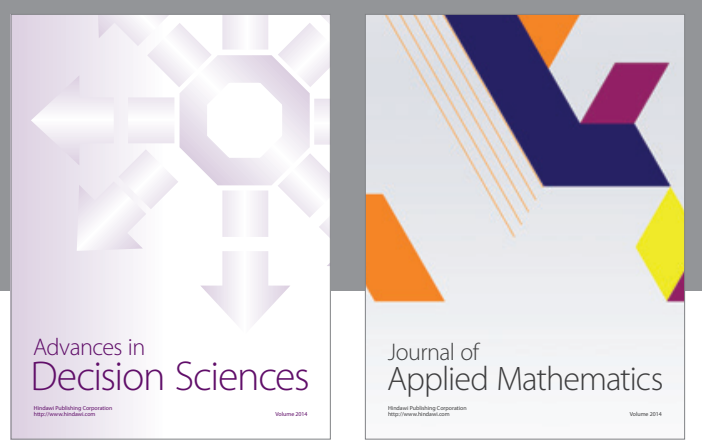

Algebra

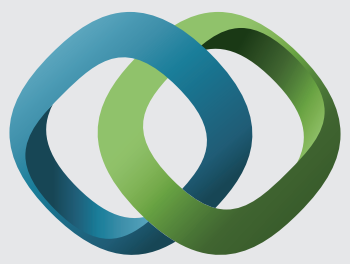

\section{Hindawi}

Submit your manuscripts at

http://www.hindawi.com
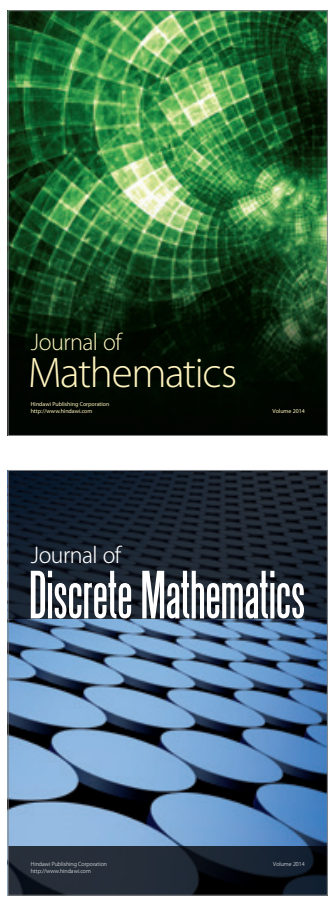

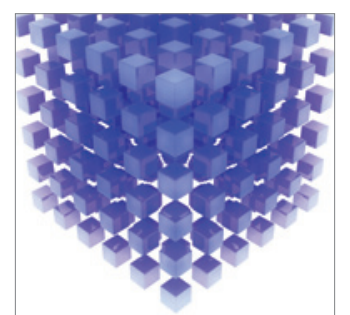

Mathematical Problems in Engineering
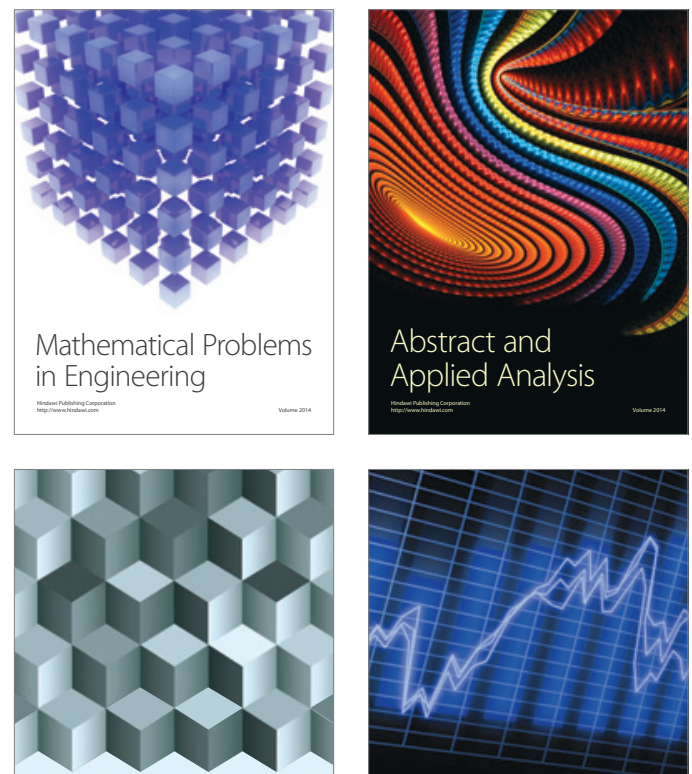

Journal of

Function Spaces

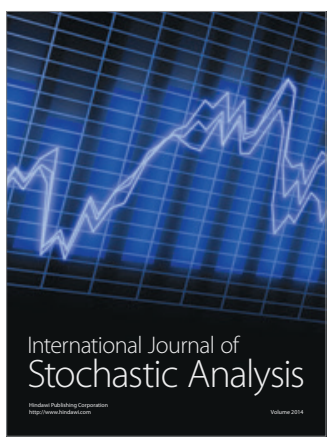

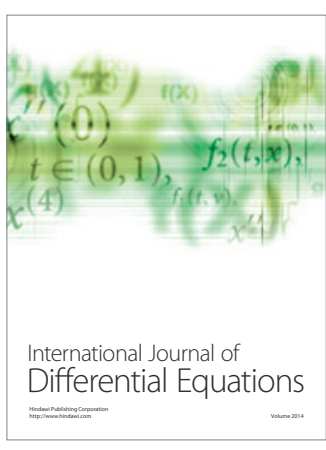
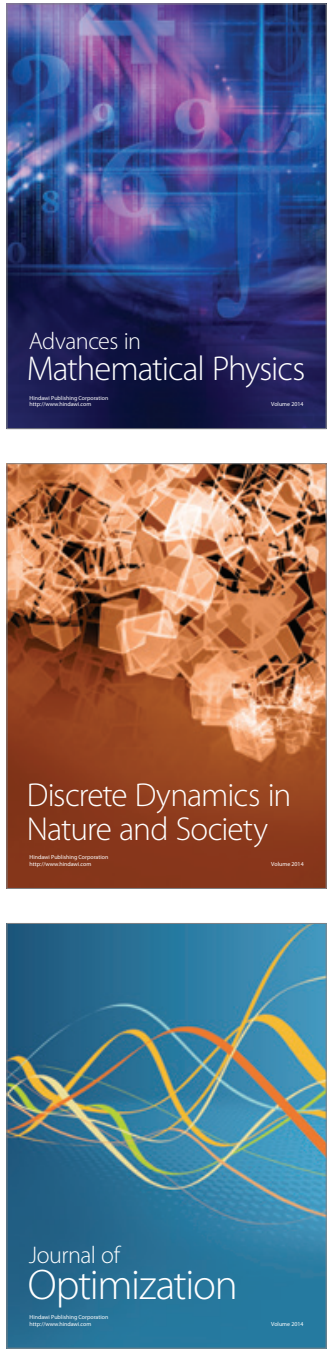